\title{
$\mu$-VALUES FOR MATRICES CORRESPONDING TO SYMMETRIES IN CONTROL SYSTEMS
}

\author{
MUTTI-UR REHMAN*, M. FAZEEL ANWAR \\ Department of Mathematics, Sukkur IBA University, 65200 Sukkur-Pakistan \\ ${ }^{*}$ Corresponding author: mutti.rehman@iba-suk.edu.pk
}

\begin{abstract}
In this article we consider numerical approximation of structured singular values ( $\mu$-values).
The lower bounds for $\mu$-values are approximated by using ordinary differential equations based technique.

The structured singular values provide a vital tool to investigate stability of feedback systems. We also compute the lower bounds of $\mu$-values for certain matrices that correspond to symmetries in control systems.
\end{abstract}

\section{INTRODUCTION}

The Structured Singular Values known as $\mu$-values is a well-known mathematical tool in control, introduced in 1981 by J. C. Doyle [13]. They can be used to discuss stability of linear systems subject to certain perturbations. Applications of structured singular values in engineering system are described in [14]. The exact computation of $\mu$-values is known to be an NP-hard problem see [2].

A considerable effort has been made to compute the lower and upper bound for structured singular values. The power method [10] provides a lower bound for $\mu$-values when we consider pure complex perturbations. It however fails to converge for pure real uncertainties for more details see [16]. The upper bound of $\mu$-values provides critical information which guarantees the stability of feedback linear systems. The well-known Matlab function mussv available in MATLAB control toolbox approximates an upper bounds for structured singular values by means of diagonal balancing and Linear Matrix Inequality techniques [5]. The methodology proposed in [12] is based on a two level algorithm, inner and outer algorithm. In inner algorithm, we attempt

Received 2018-05-09; accepted 2018-08-02; published 2018-09-05.

2010 Mathematics Subject Classification. 65F15, 34H05,

Key words and phrases. $\mu$-values; spectral radius; family of block diagonal perturbations.

(C) 2018 Authors retain the copyrights of their papers, and all open access articles are distributed under the terms of the Creative Commons Attribution License. 
to solve an optimization problem while outer algorithm allows us to compute an extremizer by varying a small parameter $\epsilon$.

In [4], Danielson used symmetric groups to design model predictive controllers with reduced complexity for constrained linear control systems. In model predictive control, the control input is obtained by solving a constrained finite time optimal control problem. For a piecewise affine control law symmetries are statespace and input-space transformations that relate controller pieces. Using symmetry he could discard some of the pieces of a given controller. These discarded pieces can also be reconstructed using symmetry. Using symmetries of the control system he was able to reduce the complexity of the controller and save memory without sacrificing performance. It was also noted that the amount of reduction in complexity depends on the number of symmetries possessed by the system. For systems with large symmetry groups the techniques presented in [4] can significantly reduce the complexity of the piecewise affine control-law produced using explicit model predictive control.The goal of this article is to compute the $\mu$-values for matrices corresponding to a control system whose symmetry group is $S_{5}$. We present a comparison between lower bounds of $\mu$-values approximated by mussv and the algorithm presented in [12].

\section{BASIC FRAMEWORK}

Let $\mathbb{C}^{n, n}\left(\mathbb{R}^{n, n}\right)$ denote the collection of $n \times n$ complex (real) matrices and let $M \in \mathbb{C}^{n, n}$. We denote a family of block diagonal matrices by

$$
\Theta_{\mathbb{B}}=\left\{\operatorname{Diag}\left(c_{i} I_{i}, \Gamma_{j}\right): c_{i} \in \mathbb{C}(\mathbb{R}), \Gamma_{j} \in \mathbb{C}^{m_{j}, m_{j}}\left(\mathbb{R}^{m_{j}, m_{j}}\right)\right\}
$$

In the above equation, $I_{i}$ is an identity matrix having dimension $i$.

Definition 2.1. [9]. The structured singular values denoted by $\mu$ for a given matrix $M \in \mathbb{C}^{n, n}$ or $M \in \mathbb{R}^{n, n}$ and a set of block diagonal matrices $\Theta_{\mathbb{B}}$ is defined as

$$
\mu_{\Theta_{\mathbb{B}}}(M):=\frac{1}{\min \left\{\|\Delta\|_{2}: \Delta \in \Theta_{\mathbb{B}}, \operatorname{det}(I-M \Delta)=0\right\}} .
$$

In above definition $2.1, \operatorname{det}(\cdot)$ represent the determinant of a matrix $(I-M \Delta)$ while minimum is over an admissible perturbation $\Delta$.

In this particular case we will denote the set of pure complex uncertainties by $\Theta_{\mathbb{B}}^{\prime}$. If $\Delta \in \Theta_{\mathbb{B}}^{\prime}$, there is a function $\exp (i \Phi) \Delta \in \Theta_{\mathbb{B}}^{\prime}$ for any real number $\Phi$ and as a consequence we have $\Delta \in \Theta_{\mathbb{B}}^{\prime}$ such that the spectral radius of $M \Delta$ attains the exact value 1 iff there is $\Delta^{*} \in \Theta_{\mathbb{B}}^{\prime}$ such that $M \Delta^{*}$ has the eigenvalue 1 . The perturbation $\Delta^{*}$ is constructed in such a way that it possesses a unit 2-norm and as $\operatorname{result} \operatorname{det}\left(I-M \Delta^{*}\right)=0$. The above construction allows us to write an alternate definition of $\mu$-values when pure complex uncertainties are under consideration. 


$$
\Theta_{\mathbb{B}}^{\prime}=\frac{1}{\min \left\{\|\Delta\|_{2}: \Delta \in \Theta_{\mathbb{B}}^{\prime}, \rho(M \Delta)=1\right\}} .
$$

In Equ. $(2.2), \rho(\cdot)$ denotes the spectral radius of a matrix $M \Delta$.

2.1. The $\mu$-value based on a structured spectral value set. For a given $n$-dimensional complex matrix $M \in \mathbb{C}^{n \times n}$ and a perturbation level $\epsilon$ the structured spectral value set is the collection of all eigenvalues of matrix $(\epsilon M \Delta)$ defined as

$$
\Lambda_{\epsilon_{0}}^{\Theta_{\mathbb{B}}}(M)=\left\{\lambda \in \Lambda(\epsilon M \Delta): \Delta \in \Theta_{\mathbb{B}}\right\}
$$

where $\Lambda(\cdot)$ denotes the spectrum of a matrix and $\|\Delta\|_{2}=1$. For mixed real and complex uncertainties, we let

$$
\Sigma_{\epsilon}^{\Theta_{\mathbb{B}}}(M)=\left\{\eta=1-\lambda_{1}: \lambda_{1} \in \Lambda_{\epsilon}^{\Theta_{\mathbb{B}}}(M)\right\} .
$$

The formulation in Equ. (2.4) allows us to write down structured singular values defined in Equ. (2.2) as follows:

$$
\mu_{\Theta_{\mathbb{B}}}(M)=\frac{1}{\arg \min \left\{0 \in \Sigma_{\epsilon}^{\Theta_{\mathbb{B}}}(M)\right\}} .
$$

While on the other hand when pure complex uncertainties are under consideration then Equ. (2.3) allows us to alternatively express $\mu$-value as

$$
\mu_{\Theta_{\mathbb{B}}^{\prime}}(M)=\frac{1}{\arg \min \left\{\max \left|\lambda_{1}\right|=1\right\}} .
$$

2.2. Mathematical Problem. We consider the following optimization problem,

$$
\xi\left(\epsilon_{0}\right)=\arg \min |\eta|
$$

where $\eta \in \Sigma_{\epsilon_{0}}^{\Theta_{\mathbb{B}}}(M)$ for some fixed parameter $\epsilon_{1}>0$. It is clear from the above discussion that the $\mu$-value $\mu_{\Theta_{\mathbb{B}}}(M)$ is the reciprocal of the minimum value of $\epsilon_{1}$ for which $\eta\left(\epsilon_{1}\right)=0$. Therefore we suggests a two-level algorithm that is inner and outer algorithm. For inner algorithm, we solve Equ. (2.7) by constructing and then solving a gradient system of ordinary differential equations. While for the case of outer algorithm, we make use of an iterative method to first vary the perturbation level $\epsilon_{1}$. This gives the knowledge of the computation of derivative of a local extremizer say $\Delta\left(\epsilon_{1}\right)$ with respect to some fixed parameter $\epsilon_{1}$.

We addressed the case of a purely complex uncertainties when $\Theta_{\mathbb{B}}^{*}$ by taking the inner algorithm in order to compute a local optimum for

$$
\lambda\left(\epsilon_{1}\right)=\arg \max \left|\lambda_{1}\right| .
$$

In Equ. (2.8), $\lambda_{1} \in \Lambda_{\epsilon_{1}}^{\Theta_{\mathbb{B}}^{*}}(M)$ which yields a lower bound for the $\mu$-value in case of pure complex perturbations that is $\mu_{\Delta_{\mathbb{B}}^{*}}(M)$. 


\section{Purely Complex uncertainties}

In this section, we give a solution of the maximization problem (2.8) for $M \in \mathbb{C}^{n, n}$ while considering the set of pure complex uncertainties given below

$$
\Theta_{\mathbb{B}}^{*}=\left\{\operatorname{diag}\left(\alpha_{1} I_{1}, \ldots, \alpha_{n} I_{n} ; \Delta_{1}, \ldots, \Delta_{F}\right): \alpha_{i} \in \mathbb{C}, \Delta_{j} \in \mathbb{C}^{m_{j}, m_{j}}\right\}
$$

The following lemma describes the behavior of the eigenvalues of a matrix valued function.

Lemma 3.1. For a family of matrices $\Upsilon: \mathbb{R} \rightarrow \mathbb{C}^{n, n}$ suppose that $\lambda_{1}(t)$ is an eigenvalue of a matrix valued function $\Upsilon(t)$ which converges to a simple eigenvalue $\lambda^{\prime}$ of $\Upsilon_{0}=\Upsilon(0)$ as $t \rightarrow 0$. Then $\lambda_{1}(t)$ is analytic near $t=0$ with

$$
\frac{d \lambda_{1}}{d t}=\frac{w_{0}^{*} \Upsilon_{1} v_{0}}{w_{0}^{*} v_{0}},
$$

where $\Upsilon_{1}=\dot{\Upsilon}(0)$ and $v_{0}, w_{0}$ are right and left eigenvectors of $\Upsilon_{0}$ associated to $\lambda^{\prime}$, that is, $\left(\Upsilon_{0}-\lambda^{\prime} I\right) v_{0}=0$ and $w_{0}^{*}\left(\Upsilon_{0}-\lambda^{\prime} I\right)=0$.

To deal with the optimization problem (2.8) we need to compute an uncertainty $\Delta_{\text {local }}$ in such a way that $\rho\left(\epsilon_{1} A \Delta_{\text {local }}\right)$ has the maximum growth along $\Delta \in \Theta_{\mathbb{B}}^{*}$ with $\|\Delta\|_{2} \leq 1$. In the following we call $\lambda_{1}$ the greatest eigenvalue if $\left|\lambda_{1}\right|$ equals to the spectral radius.

Definition 3.2. A matrix valued function $\Delta \in \Theta_{\mathbb{B}}^{*}$ such that $\|\Delta\|$ possesses a unit 2-norm and $\left(\epsilon_{1} M \Delta\right)$ has greatest eigenvalue which maximizes the modulus of $\Lambda_{\epsilon_{1}}^{\Theta_{\mathbb{B}}^{*}}(M)$ is called a local extremizer. In the following theorem 3.3, we give the characterization of local extremizers towards a gradient system of ordinary differential equations.

Theorem 3.3 [12]. Let

$$
\Delta_{\text {local }}=\operatorname{Diag}\left(\alpha_{1} I_{1}, \ldots, \alpha_{n} I_{n} ; \Delta_{1}, \ldots, \Delta_{F}\right)
$$

In above equation the $\Delta_{\text {local }}$ possesses a unit 2-norm and is a local extremizer of $\Lambda_{\epsilon_{1}}^{\Theta_{\mathbb{B}}^{*}}(A)$. Further suppose that the matrix $\left(\epsilon_{1} M \Delta_{\text {local }}\right)$ possesses a simple greatest eigenvalue that is $\lambda_{1}=\left|\lambda_{1}\right| e^{i \theta}$, with the right and left eigenvectors $v$ and $w$ which are scaled so that $s=e^{i \theta} w^{*} v>0$. partitioning of $u$ and $v$ yields

$$
\begin{gathered}
v=\left(v_{1}^{\mathrm{T}}, \ldots, v_{n}^{\mathrm{T}}, v_{n+1}^{\mathrm{T}}, \ldots, v_{n+F}^{\mathrm{T}}\right)^{\mathrm{T}} \\
u=A^{*} w=\left(u_{1}^{\mathrm{T}}, \ldots, u_{n}^{\mathrm{T}}, u_{n+1}^{\mathrm{T}}, \ldots, u_{n+F}^{\mathrm{T}}\right)^{\mathrm{T}},
\end{gathered}
$$

additionally we assume that

$$
\begin{aligned}
& u_{k}^{*} v_{k} \neq 0 \quad \forall k=1, \ldots, n, \\
& \left\|u_{n+h}\right\|_{2} \cdot\left\|v_{n+h}\right\|_{2} \neq 0 \quad \forall h=1, \ldots, F .
\end{aligned}
$$

Then

$$
\left|s_{k}\right|=1 \quad \forall k=1, \ldots, n \quad \text { and } \quad\left\|\Delta_{h}\right\|_{2}=1 \quad \forall h=1, \ldots, F
$$


3.1. System of ODEs to compute extremal points of $\Lambda_{\epsilon}^{\Delta_{\mathbb{B}}^{*}}(M)$. We now compute a local maximizer for $\left|\lambda_{1}\right|$ where $\lambda_{1} \in \Lambda_{\epsilon_{1}}^{\Theta_{\mathbb{B}}}(M)$. For this we first construct a matrix valued function $\Delta(t)$ in such a way that the greatest eigenvalue $\lambda_{(t)}$ of the matrix $\left(\epsilon_{1} M \Delta(t)\right)$ has the maximum growth. We then derive and solve a gradient system of ordinary differential equations which satisfies the initial choice $\Delta(0)$.

3.2. The local optimization problem. Let $\lambda_{1}=\left|\lambda_{1}\right| e^{i \theta}$ be a simple eigenvalue. Further suppose that $v, w$ are normalized so that

$$
\|w\|=\|v\|=1, \quad w^{*} v=\left|w^{*} v\right| e^{-i \theta} .
$$

By making use of Lemma 3.1, we have

$$
\frac{d}{d t}\left|\lambda_{1}\right|^{2}=2\left|\lambda_{1}\right| \operatorname{Re}\left(\frac{u^{*} \dot{\Delta} v}{e^{i \theta} w^{*} v}\right)=\frac{2\left|\lambda_{1}\right|}{\left|w^{*} v\right|} \operatorname{Re}\left(u^{*} \dot{\Delta} v\right)
$$

where $u=M^{*} w$. For $\Delta \in \Theta_{\mathbb{B}}$ we aim to compute the direction $\dot{\Delta}=\tau$ that maximizes the local growth of the modulus of $\lambda_{1}$. We get

$$
\tau=\operatorname{diag}\left(\omega_{1} I_{r_{1}}, \ldots, \omega_{s} I_{r_{N}}, \Omega_{1}, \ldots, \Omega_{F}\right)
$$

as a solution of the optimization problem

$$
\tau_{*}=\arg \max \left\{\operatorname{Re}\left(u^{*} \tau x\right)\right\}
$$

$$
\text { subject to } \operatorname{Re}\left(\bar{\delta}_{i} \omega_{i}\right)=0, \quad i=1: N
$$

$$
\text { and } \operatorname{Re}\left\langle\Delta_{j}, \Omega_{j}\right\rangle=0, j=1: F \text {. }
$$

In the following Lemma 3.2.1, we give the solution $\tau_{*}$ of the optimization problem as discussed in the (3.8).

Lemma 3.2.1 [12].

$$
\tau_{*}=\operatorname{Diag}\left(\omega_{1} I_{r_{1}}, \ldots, \omega_{N} I_{r_{N}}, \Omega_{1}, \ldots, \Omega_{F}\right)
$$

with

$$
\begin{aligned}
\omega_{i} & =\nu_{i}\left(v_{i}^{*} u_{i}-\operatorname{Re}\left(v_{i}^{*} u_{i} \bar{s}_{i}\right) s_{i}\right), \quad i=1, \ldots, N \\
\Omega_{j} & =\zeta_{j}\left(u_{N+j} v_{N+j}^{*}-\operatorname{Re}\left\langle\Delta_{j}, u_{N+j} v_{N+j}^{*}\right\rangle \Delta_{j}\right), \quad j=1, \ldots, F .
\end{aligned}
$$

The coefficient $\nu_{i}>0$ is the reciprocal of the absolute value of the expression appearing in the right-hand side in Equ. (3.10) when it's different from zero and $\nu_{i}=1$ else. While the coefficient $\zeta_{j}>0$ is the reciprocal of the Frobenius norm of the matrix appearing in the right hand side of Equ. (3.11) if it's different from zero and $\zeta_{j}=1$ else.

We write down the result as obtained in the previous Lemma 1.2 as:

$$
\tau_{*}=G_{1} P_{\Theta_{\mathbb{B}}^{*}}\left(u v^{*}\right)-D_{2} \Delta
$$


In above equation $P_{\Theta_{\mathbb{B}}^{*}}(\cdot)$ is the orthogonal projection while $G_{1}, D_{2} \in \Theta_{\mathbb{B}}^{*}$ are diagonal matrices while the matrix $D_{1}$ is positive.

3.3. The gradient system of ordinary differential equations. The result in the previous Lemma 3.2 .1 allows us to consider the following differential equation on $\Theta_{\mathbb{B}}^{*}$ :

$$
\dot{\Delta}=G_{1} P_{\Theta_{\mathbb{B}}^{*}}\left(u v^{*}\right)-D_{2} \Delta .
$$

In the above equation $v(t)$ is an eigenvector having the unit 2-norm ans is associated to a simple eigenvalue $\lambda_{(t)}$ of the matrix $\left(\epsilon_{1} M \Delta(t)\right)$ for some fixed parameter $\epsilon_{1}>0$. The differential equation (3.13) is a gradient system of ordinary differential equations because it's the right-hand side is the projected gradient of $\tau \mapsto R e\left(u^{*} \tau v\right)$.

3.4. Choice of initial value matrix and $\epsilon_{0}$. In order to compute $\epsilon_{0}$ we choose the initial value matrix

$$
\Delta_{0}=D P_{\Delta_{\mathbb{B}}}\left(w v^{*}\right)
$$

where $D$ is the positive diagonal matrix such that $\Delta_{0} \in \Theta_{\mathbb{B}}$. As a natural choice for the initialization of the perturbation level, we take $\epsilon_{0}$ as

$$
\epsilon_{0}=\frac{1}{\widehat{\mu}_{\Theta_{\mathbb{B}}}(M)} .
$$

where $\widehat{\mu}_{\Theta_{\mathbb{B}}}(M)$ is the upper bound of $\mu$-value approximated by mussv.

\section{Numerical Experimentation}

In this section, we present the main contribution which is the numerical approximation of both lower and upper bounds of $\mu$-values. These results are computed by well-known Matlab routine mussv and the algorithm [12].

Example 1. In table 1, we give comparison of numerical approximation to both lower and upper bounds of structured singular values approximated by the well-known MATLAB routine mussv and algorithm [12] for the matrix $A_{4}$. The matrix $A_{4}$ is gievn as below. In first column of table 1 , we present the size of the matrix $A_{4}$. While in the next column, we present the family of block diagonal matrices which is denoted by $\Theta_{\mathbb{B}}$. In the third, fourth and fifth columns, we present both upper and lower bounds of SSV, that is, $\mu_{u}^{m u s s v}$, $\mu_{l}^{\text {mussv }}$ approximated by MATLAB routine mussv and the lower bound $\mu_{l}^{N e w}$ approximated by algorithm [12] respectively.

$$
A_{4}=\left[\begin{array}{cccc}
-0.5+1.4434 i & -0.5774 i & 0.5-0.2887 i & -0.5+0.2887 \\
-0.5+0.8660 i & 0 & 0 & 0 \\
-0.5+0.8660 i & 0 & -0.5-0.8660 i & -1 \\
-0.5-0.2887 i & -0.5774 i & 0.5774 i & -0.8660 i
\end{array}\right]
$$




\begin{tabular}{||l||c||c||c||c||}
\hline$n$ & $\Theta_{\mathbb{B}}$ & $\mu_{u}^{\text {mussv }}$ & $\mu_{l}^{\text {mussv }}$ & $\mu_{l}^{\text {New }}$ \\
\hline 04 & $\left\{\operatorname{diag}\left(\Delta_{1}\right): \Delta_{1} \in \mathbb{C}^{4,4}\right\}$ & 2.5031 & 2.5030 & 2.5030 \\
\hline 04 & $\left\{\operatorname{diag}\left(\delta_{1} I_{1}, \delta_{2} I_{1}, \delta_{3} I_{1}, \delta_{4} I_{1}\right): \delta_{1}, \delta_{2}, \delta_{3}, \delta_{4} \in \mathbb{R}\right\}$ & 0.6354 & 0.0000 & 0.6297 \\
\hline 04 & $\left\{\operatorname{diag}\left(\delta_{1} I_{1}, \delta_{2} I_{1}, \delta_{3} I_{1}, \delta_{4} I_{1}\right): \delta_{1}, \delta_{2}, \delta_{3}, \delta_{4} \in \mathbb{C}\right\}$ & 2.3780 & 2.3748 & 2.3748 \\
\hline 04 & $\left\{\operatorname{diag}\left(\delta_{1} I_{1}, \delta_{2} I_{1}, \Delta_{2}\right): \delta_{1}, \delta_{2} \in \mathbb{R}, \Delta_{1} \in \mathbb{C}^{2,2}\right\}$ & 1.8114 & 1.7568 & 1.7552 \\
\hline 04 & $\left\{\operatorname{diag}\left(\delta_{1} I_{1}, \delta_{2} I_{1}, \Delta_{2}\right): \delta_{1}, \delta_{2} \in \mathbb{C}, \Delta_{1} \in \mathbb{C}^{2,2}\right\}$ & 2.3832 & 2.3813 & 2.3811 \\
\hline 04 & $\left\{\operatorname{diag}\left(\Delta_{1}, \Delta_{2}\right): \Delta_{1}, \Delta_{2} \in \mathbb{C}^{2,2}\right\}$ & 2.4047 & 2.4027 & 2.4029 \\
\hline 04 & $\left\{\operatorname{diag}\left(\delta_{1} I_{1}, \Delta_{2}\right): \delta_{1} \in \mathbb{R}, \Delta_{2} \in \mathbb{C}^{3,3}\right\}$ & 1.8415 & 1.8415 & 1.8414 \\
\hline
\end{tabular}

TABLE 1. Computation of bounds of $\mu$-values

\begin{tabular}{|c|c|c|c|c|}
\hline$n$ & $\Theta_{\mathbb{B}}$ & $\mu_{u}^{m u s s v}$ & $\mu_{l}^{\text {mussv }}$ & $\mu_{l}^{N e w}$ \\
\hline 04 & $\left\{\operatorname{diag}\left(\Delta_{1}\right): \Delta_{1} \in \mathbb{C}^{4,4}\right\}$ & 2.8765 & 2.8765 & 2.8763 \\
\hline 04 & $\left\{\operatorname{diag}\left(\delta_{1} I_{1}, \delta_{2} I_{1}, \delta_{3} I_{1}, \delta_{4} I_{1}\right): \delta_{1}, \delta_{2}, \delta_{3}, \delta_{4} \in \mathbb{R}\right\}$ & 1.5023 & 0.0000 & 1.0000 \\
\hline 04 & $\left\{\operatorname{diag}\left(\delta_{1} I_{1}, \delta_{2} I_{1}, \delta_{3} I_{1}, \delta_{4} I_{1}\right): \delta_{1}, \delta_{2}, \delta_{3}, \delta_{4} \in \mathbb{C}\right\}$ & 2.7375 & 2.7326 & 2.7326 \\
\hline 04 & $\left\{\operatorname{diag}\left(\delta_{1} I_{1}, \delta_{2} I_{1}, \Delta_{2}\right): \delta_{1}, \delta_{2} \in \mathbb{R}, \Delta_{1} \in \mathbb{C}^{2,2}\right\}$ & 1.8732 & 1.8716 & 0.5373 \\
\hline 04 & $\left\{\operatorname{diag}\left(\delta_{1} I_{1}, \delta_{2} I_{1}, \Delta_{2}\right): \delta_{1}, \delta_{2} \in \mathbb{C}, \Delta_{1} \in \mathbb{C}^{2,2}\right\}$ & 2.7375 & 2.7334 & 2.7336 \\
\hline 04 & $\left\{\operatorname{diag}\left(\Delta_{1}, \Delta_{2}\right): \Delta_{1}, \Delta_{2} \in \mathbb{C}^{2,2}\right\}$ & 2.7375 & 2.7373 & 2.7372 \\
\hline
\end{tabular}

TABLE 2. Computation of bounds of $\mu$-values

In table 2, we give comparison of numerical approximation to both lower and upper bounds of structured singular values approximated by the well-known MATLAB routine mussv and algorithm [12] for the matrix $B_{4}$. The matrix $B_{4}$ is gievn as below. In first column of table 2 , we present the size of the matrix $B_{4}$. While in the next column, we present the family of block diagonal matrices which is denoted by $\Theta_{\mathbb{B}}$. In the third, fourth and fifth columns we present both upper and lower bounds that is $\mu_{u}^{m u s s v}, \mu_{l}^{\text {mussv }}$ approximated by MATLAB routine mussv and the lower bound $\mu_{l}^{N e w}$ approximated by algorithm [12] respectively.

$$
B_{4}=\left[\begin{array}{cccc}
0.5+0.8660 i & -1 & -0.5-0.8660 i & -1 \\
-0.5+0.8660 i & 0 & -0.5-0.8660 i & -1 \\
0.5774 i & -0.5+0.2887 i & 0.5-0.2887 i & -0.5+0.2887 i \\
-0.5+0.2887 i & -0.5774 i & 0.5-0.2887 i & 1-0.5774 i
\end{array}\right]
$$


Example 2. Consider the following four dimensional matrix $A_{5}$.

$$
A_{5}=\left[\begin{array}{cccc}
-0.5+0.8660 i & 0.2500-0.4330 i & 0.5 & 0.5 \\
0 & -0.75-0.4330 i & -0.5000 & 0+0.8660 i \\
0.5+0.8660 i & 0 & 0 & 0 \\
-0.5-0.8660 i & 0.25+0.4330 i & 0.25+1.2990 i & 0.25-0.4330 i
\end{array}\right]
$$

Consider the perturbations set as

$$
\Theta_{\mathbb{B}}=\left\{\operatorname{diag}\left(\delta_{1} I_{1}, \delta_{2} I_{1}, \delta_{3} I_{1}, \delta_{4} I_{1}\right): \delta_{1}, \delta_{2}, \delta_{3}, \delta_{4} \in \mathbb{R}\right\} .
$$

By applying MATLAB function mussv, we have obtained the perturbation $\widehat{\Delta}$ with

$$
\widehat{\Delta}=1.0 e+050\left[\begin{array}{llll}
4.9136 & 0.0000 & 0.0000 & 0.0000 \\
0.0000 & 4.9136 & 0.0000 & 0.0000 \\
0.0000 & 0.0000 & 4.9136 & 0.0000 \\
0.0000 & 0.0000 & 0.0000 & 4.9136
\end{array}\right]
$$

while $\|\widehat{\Delta}\|_{2}=4.9136 e+050$. For this particular example, we have obtained an upper bound $\mu_{P D}^{\text {upper }}=1.5797$ while the same lower bound as $\mu_{P D}^{\text {lower }}=0.0000$. By making use of our algorithm [12], we have obtained the perturbation $\epsilon^{*} \Delta^{*}$ with

$$
\Delta^{*}=\left[\begin{array}{cccc}
-0.7049 & 0.0000 & 0.0000 & 0.0000 \\
0.0000 & -1.0000 & 0.0000 & 0.0000 \\
0.0000 & 0.0000 & -1.0000 & 0.0000 \\
0.0000 & 0.0000 & 0.0000 & 0.7490
\end{array}\right]
$$

and $\epsilon^{*}=0.7549$. while $\left\|\Delta^{*}\right\|_{2}=1$. The same lower bound can be obtained $\mu_{\text {New }}^{\text {lower }}=1.3248$ as the one obtained by mussv.

In the table 3, we give comparison of numerical approximation to both lower and upper bounds of structured singular values approximated by the well-known MATLAB routine mussv and algorithm [12] for the matrix $A_{5}$. The matrix $A_{5}$ is gievn as below. In first column of table 3 , we present the size of the matrix $A_{5}$. While in the next column, we present the family of block diagonal matrices which is denoted by $\Theta_{\mathbb{B}}$. In the third, fourth and fifth columns we present both upper and lower bounds that is $\mu_{u}^{\text {mussv }}, \mu_{l}^{\text {mussv }}$ approximated by MATLAB routine mussv and the lower bound $\mu_{l}^{\text {New }}$ approximated by algorithm [12] respectively.

In the following table 4, we give comparison of numerical approximation to both lower and upper bounds of structured singular values approximated by the well-known MATLAB routine mussv and algorithm [12] for the matrix $B_{5}$. The matrix $B_{5}$ is gievn as below. In first column of table 4 , we present the size of the matrix $B_{5}$. While in the next column, we present the family of block diagonal matrices which is denoted by $\Theta_{\mathbb{B}}$. In the third, fourth and fifth columns we present both upper and lower bounds that is 


\begin{tabular}{||c||c||c||c||c||}
\hline$n$ & $\Theta_{\mathbb{B}}$ & $\mu_{u}^{\text {mussv }}$ & $\mu_{l}^{\text {mussv }}$ & $\mu_{l}^{\text {New }}$ \\
\hline 04 & $\left\{\operatorname{diag}\left(\Delta_{1}\right): \Delta_{1} \in \mathbb{C}^{4,4}\right\}$ & 2.2701 & 2.2701 & 2.2699 \\
\hline 04 & $\left\{\operatorname{diag}\left(\delta_{1} I_{1}, \delta_{2} I_{1}, \delta_{3} I_{1}, \delta_{4} I_{1}\right): \delta_{1}, \delta_{2}, \delta_{3}, \delta_{4} \in \mathbb{C}\right\}$ & 1.8679 & 1.8679 & 1.8675 \\
\hline 04 & $\left\{\operatorname{diag}\left(\delta_{1} I_{1}, \delta_{2} I_{1}, \Delta_{1}\right): \delta_{1}, \delta_{2} \in \mathbb{C}, \Delta_{1} \in \mathbb{C}^{2,2}\right\}$ & 2.0084 & 2.0084 & 2.0076 \\
\hline 04 & $\left\{\operatorname{diag}\left(\delta_{1} I_{1}, \delta_{2} I_{1}, \Delta_{1}\right): \delta_{1}, \delta_{2} \in \mathbb{R}, \Delta_{1} \in \mathbb{C}^{2,2}\right\}$ & 2.1863 & 2.1863 & 2.1861 \\
\hline 04 & $\left\{\operatorname{diag}\left(\delta_{1} I_{1}, \Delta_{1}\right): \delta_{1} \in \mathbb{R}, \Delta_{1} \in \mathbb{C}^{3,3}\right\}$ & 2.0242 & 2.0242 & 0.5000 \\
\hline 04 & $\left\{\operatorname{diag}\left(\delta_{1} I_{1}, \Delta_{1}\right): \delta_{1} \in \mathbb{C}, \Delta_{1} \in \mathbb{C}^{3,3}\right\}$ & 2.1956 & 2.1932 & 2.1934 \\
\hline
\end{tabular}

TABLE 3. Computation of bounds of $\mu$-values

\begin{tabular}{|c|c|c|c|c|}
\hline$n$ & $\Delta_{\mathbb{B}}$ & $\mu_{u}^{m u s s v}$ & $\mu_{l}^{\text {mussv }}$ & $\mu_{l}^{N e w}$ \\
\hline 04 & $\left\{\operatorname{diag}\left(\Delta_{1}\right): \Delta_{1} \in \mathbb{C}^{4,4}\right\}$ & 2.9458 & 2.9458 & 2.9456 \\
\hline 04 & $\left\{\operatorname{diag}\left(\delta_{1} I_{1}, \delta_{2} I_{1}, \delta_{3} I_{1}, \delta_{4} I_{1}\right): \delta_{1}, \delta_{2}, \delta_{3}, \delta_{4} \in \mathbb{R}\right\}$ & 1.5155 & 0.0000 & 1.0000 \\
\hline 04 & $\left\{\operatorname{diag}\left(\delta_{1} I_{2}, \delta_{2} I_{2}\right): \delta_{1}, \delta_{2} \in \mathbb{R}\right\}$ & 1.2164 & 0.0000 & 1.0894 \\
\hline 04 & $\left\{\operatorname{diag}\left(\delta_{1} I_{1}, \Delta_{2}\right): \delta_{1} \in \mathbb{R}, \Delta_{2} \in \mathbb{R}^{3,3}\right\}$ & 1.1641 & 0.0000 & 1.0000 \\
\hline 04 & $\left\{\operatorname{diag}\left(\Delta_{1}\right): \Delta_{1} \in \mathbb{R}^{4,4}\right\}$ & 1.2165 & 0.0000 & 0.7275 \\
\hline
\end{tabular}

TABLE 4. Computation of bounds of $\mu$-values

$\mu_{u}^{\text {mussv }}, \mu_{l}^{\text {mussv }}$ approximated by MATLAB routine mussv and the lower bound $\mu_{l}^{N e w}$ approximated by algorithm [12] respectively.

$$
B_{5}=\left[\begin{array}{cccc}
-1 & 0.75-0.4330 i & 0.25+1.2990 i & -0.75-0.4330 i \\
0 & -0.5 & 0.25+1.2990 i & -0.25-1.2990 i \\
0 & -0.8660 i & -0.2500-1.2990 i & -0.75+0.4330 i \\
0 & 0.75+0.4330 i & -0.75-0.4330 i & -0.2500+0.4330 i
\end{array}\right] .
$$

Example 3. Consider the following four dimensional matrix $A_{6}$.

$$
A_{6}=\left[\begin{array}{cccccc}
0.3819 & 0.3819 & 1.0000 & 0 & 0 & 0 \\
1.2360 & -0.3819 & 0.1680 & 0 & 0 & 0 \\
-0.6180 & 1.0000 & -0.6180 & 0 & 0 & 0 \\
0 & 0 & 0 & 0.3819 & -1.2360 & 0.3819 \\
0 & 0 & 0 & 1.2360 & -0.3819 & 0.6180 \\
0 & 0 & 0 & -1.0000 & 0.6180 & 0.6180
\end{array}\right],
$$

Consider the perturbation set as 


\begin{tabular}{||l||c||c||c||c||}
\hline$n$ & $\Delta_{\mathbb{B}}$ & $\mu_{u}^{\text {mussv }}$ & $\mu_{l}^{\text {mussv }}$ & $\mu_{l}^{\text {New }}$ \\
\hline 06 & $\left\{\operatorname{diag}\left(\delta_{1} I_{6}\right): \delta_{1} \in \mathbb{C}\right\}$ & 1.3061 & 1.3037 & 1.3037 \\
\hline 06 & $\left.\left\{\operatorname{diag}\left(\delta_{1} I_{6}\right): \delta_{1} \in \mathbb{R}\right\}\right\}$ & 0.9560 & 0.0000 & 0.9560 \\
\hline 06 & $\left\{\operatorname{diag}\left(\delta_{i} I_{i}\right): \delta_{i} \in \mathbb{R}, \forall i=1: 6\right\}$ & 1.9330 & 1.9330 & 1.9330 \\
\hline 06 & $\left\{\operatorname{diag}\left(\delta_{i} I_{i}\right): \delta_{i} \in \mathbb{C}, \forall i=1: 6\right\}$ & 1.1875 & 0.0000 & 1.1875 \\
\hline 06 & $\left\{\operatorname{diag}\left(\delta_{1} I_{3}, \delta_{2} I_{3}\right): \delta_{1}, \delta_{2} \in \mathbb{R}\right\}$ & 0.9560 & 0.0000 & 0.9560 \\
\hline
\end{tabular}

TABLE 5. Computation of bounds of $\mu$-values

$$
\Theta_{\mathbb{B}}=\left\{\operatorname{diag}\left(\Delta_{1}\right): \Delta_{1} \in \mathbb{C}^{6,6}\right\}
$$

By applying the MATLAB function mussv, we have obtained the perturbation $\widehat{\Delta}$ with

$$
\widehat{\Delta}=\left[\begin{array}{cccccc}
0 & 0 & 0 & 0 & 0 & 0 \\
0 & 0 & 0 & 0 & 0 & 0 \\
0 & 0 & 0 & 0 & 0 & 0 \\
0 & 0 & 0 & 0.2136 & 0.2414 & -0.2030 \\
0 & 0 & 0 & -0.1765 & -0.1994 & 0.1678 \\
0 & 0 & 0 & 0.0386 & 0.0436 & -0.0367
\end{array}\right]
$$

while $\|\widehat{\Delta}\|_{2}=0.4989$. For this particular example, we have obtained an upper bound $\mu_{P D}^{\text {upper }}=2.0043$ while the same lower bound as $\mu_{P D}^{\text {lower }}=2.0043$. By making use of our algorithm [12], we have obtained the perturbation $\epsilon^{*} \Delta^{*}$ with

$$
\Delta^{*}=\left[\begin{array}{cccccc}
0 & 0 & 0 & 0 & 0 & 0 \\
0 & 0 & 0 & 0 & 0 & 0 \\
0 & 0 & 0 & 0 & 0 & 0 \\
0 & 0 & 0 & 0.4282 & 0.4837 & -0.4069 \\
0 & 0 & 0 & -0.3538 & -0.3997 & 0.3363 \\
0 & 0 & 0 & 0.0773 & 0.0874 & -0.0735
\end{array}\right]
$$

and $\epsilon^{*}=0.4989$. while $\left\|\Delta^{*}\right\|_{2}=1$. The same lower bound can be obtained $\mu_{\text {New }}^{\text {lower }}=2.0043$ as the one obtained by mussv.

In table 5, we give comparison of numerical approximation to both lower and upper bounds of structured singular values approximated by the well-known MATLAB routine mussv and algorithm [12] for the matrix $A_{6}$. The matrix $A_{6}$ is gievn as below. In first column of table 5 , we present the size of the matrix $A_{6}$. While in the next column, we present the family of block diagonal matrices which is denoted by $\Theta_{\mathbb{B}}$. In the third, fourth and fifth columns we present both upper and lower bounds that is $\mu_{u}^{\text {mussv }}, \mu_{l}^{\text {mussv }}$ approximated by MATLAB routine mussv and the lower bound $\mu_{l}^{\text {New }}$ approximated by algorithm [12] respectively. 
Consider the following four dimensional matrix $B_{6}$.

$$
B_{6}=\left[\begin{array}{cccccc}
0 & 0 & 0 & -1.0000 & 0 & 0 \\
0 & 0 & 0 & 0 & -1.0000 & 0 \\
0 & 0 & 0 & 0.3819 & 0.3819 & 1.0000 \\
-1.0000 & 0 & 0 & 0 & 0 & 0 \\
0 & -1.0000 & 0 & 0 & 0 & 0 \\
0.3819 & 0.3819 & 1.0000 & 0 & 0 & 0
\end{array}\right]
$$

Consider the perturbation set as

$$
\Theta_{\mathbb{B}}=\left\{\operatorname{diag}\left(\Delta_{1}\right): \Delta_{1} \in \mathbb{C}^{6,6}\right\}
$$

By applying MATLAB function mussv, we have obtained the perturbation $\widehat{\Delta}$ with

$$
\widehat{\Delta}=\left[\begin{array}{cccccc}
0 & 0 & 0 & 0 & 0 & 0 \\
0 & 0 & 0 & 0 & 0 & 0 \\
0 & 0 & 0 & 0 & 0 & 0 \\
0 & 0 & 0 & 0.2136 & 0.2414 & -0.2030 \\
0 & 0 & 0 & -0.1765 & -0.1994 & 0.1678 \\
0 & 0 & 0 & 0.0386 & 0.0436 & -0.0367
\end{array}\right]
$$

and $\|\widehat{\Delta}\|_{2}=0.7658$. For this example, one can obtain the upper bound $\mu_{P D}^{u p p e r}=1.3059$ while the same lower bound as $\mu_{P D}^{\text {lower }}=1.3059$. By making use of our algorithm [12], we have obtained the perturbation $\epsilon^{*} \Delta^{*}$ with

$$
\Delta^{*}=\left[\begin{array}{cccccc}
0 & 0 & 0 & -0.1848 & -0.1848 & 0.3413 \\
0 & 0 & 0 & -0.1848 & -0.1848 & 0.3413 \\
0 & 0 & 0 & -0.2002 & -0.2002 & 0.3696 \\
0 & 0 & 0 & 0 & 0 & 0 \\
0 & 0 & 0 & 0 & 0 & 0 \\
0 & 0 & 0 & 0 & 0 & 0
\end{array}\right]
$$

and $\epsilon^{*}=0.7658$. while $\left\|\Delta^{*}\right\|_{2}=1$. The same lower bound can be obtained $\mu_{N e w}^{\text {lower }}=1.3059$ as the one obtained by mussv.

In the following table 6 , we give comparison of numerical approximation to both lower and upper bounds of structured singular values approximated by the well-known MATLAB routine mussv and algorithm [12] for the matrix $B_{6}$. The matrix $B_{6}$ is gievn as below. In first column of table 6 , we present the size of the matrix $B_{6}$. While in the next column, we present the family of block diagonal matrices which is denoted by $\Theta_{\mathbb{B}}$. In the third, fourth and fifth columns we present both upper and lower bounds that is $\mu_{u}^{\text {mussv }}, \mu_{l}^{\text {mussv }}$ 


\begin{tabular}{||l||c||c||c||c||}
\hline$n$ & $\Theta_{\mathbb{B}}$ & $\mu_{u}^{\text {mussv }}$ & $\mu_{l}^{\text {mussv }}$ & $\mu_{l}^{\text {New }}$ \\
\hline 06 & $\left\{\operatorname{diag}\left(\delta_{1} I_{6}\right): \delta_{1} \in \mathbb{C}\right\}$ & 1.0016 & 1.0000 & 0.5000 \\
\hline 06 & $\left.\left\{\operatorname{diag}\left(\delta_{1} I_{6}\right): \delta_{1} \in \mathbb{R}\right\}\right\}$ & 0.9560 & 0.0000 & 1.0000 \\
\hline 06 & $\left\{\operatorname{diag}\left(\delta_{i} I_{i}\right): \delta_{i} \in \mathbb{R}, \forall i=1: 6\right\}$ & 1.0000 & 0.0000 & 1.0000 \\
\hline 06 & $\left\{\operatorname{diag}\left(\delta_{i} I_{i}\right): \delta_{i} \in \mathbb{C}, \forall i=1: 6\right\}$ & 1.0041 & 1.0000 & 0.7453 \\
\hline 06 & $\left\{\operatorname{diag}\left(\delta_{1} I_{3}, \delta_{2} I_{3}\right): \delta_{1}, \delta_{2} \in \mathbb{R}\right\}$ & 1.0813 & 0.0000 & 1.0000 \\
\hline \multicolumn{4}{|c|}{ TABLE 6. Computation of bounds of $\mu$-values }
\end{tabular}

TABLE 6. Computation of bounds of $\mu$-values

approximated by MATLAB routine mussv and the lower bound $\mu_{l}^{\text {New }}$ approximated by algorithm [12] respectively.

Example 4. In the following example, we consider a five dimensional complex matrix $A_{7}$ given as,

$$
A_{7}=\left[\begin{array}{ccccc}
0 & 1 & 0 & 1 & 1 \\
0 & 0 & -1 & -1 & -1 \\
0 & 1 & 0 & 0 & 0 \\
1 & 0 & 0 & 0 & 0 \\
0 & 0 & 0 & 1 & 0
\end{array}\right]
$$

Consider the perturbation set as

$$
\Theta_{\mathbb{B}}=\left\{\operatorname{diag}\left(\Delta_{1}\right): \Delta_{1} \in \mathbb{C}^{5,5}\right\}
$$

By applying Apply the MATLAB function mussv, we have obtained the perturbation $\widehat{\Delta}$ with

$$
\widehat{\Delta}=\left[\begin{array}{ccccc}
-0.0000 & 0.0000 & -0.0000 & 0.0000 & -0.0000 \\
0.1028 & -0.0978 & 0.0226 & -0.0000 & 0.0441 \\
0.0802 & -0.0763 & 0.0176 & -0.0000 & 0.0344 \\
0.2006 & -0.1909 & 0.0441 & -0.0000 & 0.0860 \\
0.1644 & -0.1565 & 0.0361 & -0.0000 & 0.0705
\end{array}\right]
$$

and $\|\widehat{\Delta}\|_{2}=0.4244$. For this example, one can obtain the upper bound $\mu_{P D}^{u p p e r}=2.3563$ while the same lower bound as $\mu_{P D}^{\text {lower }}=2.3563$. By making use of our algorithm [12], we have obtained the perturbation $\epsilon^{*} \Delta^{*}$ 
with

$$
\Delta^{*}=\left[\begin{array}{lllll}
0.0000 & -0.0000 & 0.0000 & 0.0000 & 0.0000 \\
0.2421 & -0.2304 & 0.0532 & 0.0000 & 0.1038 \\
0.1889 & -0.1798 & 0.0415 & 0.0000 & 0.0810 \\
0.4726 & -0.4498 & 0.1038 & 0.0000 & 0.2026 \\
0.3874 & -0.3687 & 0.0851 & 0.0000 & 0.1661
\end{array}\right]
$$

and $\epsilon^{*}=0.4244$ while $\left\|\Delta^{*}\right\|_{2}=1$ The same lower bound can be obtained $\mu_{\text {New }}^{\text {lower }}=2.3563$ as the one obtained by mussv.

In the following example, we consider a five dimensional complex matrix $B_{7}$ given as,

$$
B_{7}=\left[\begin{array}{ccccc}
1 & 0 & 0 & 0 & 0 \\
-1 & -1 & 0 & -1 & 0 \\
0 & 0 & 0 & 0 & 1 \\
0 & 0 & 0 & 1 & 0 \\
0 & 0 & 1 & 0 & 0
\end{array}\right]
$$

Consider the perturbation set as

$$
\Delta_{\mathbb{B}}=\left\{\operatorname{diag}\left(\Delta_{1}\right): \Delta_{1} \in \mathbb{C}^{5,5}\right\}
$$

By applying MATLAB function mussv, we have obtained the perturbation $\widehat{\Delta}$ with

$$
\widehat{\Delta}=\left[\begin{array}{lllll}
0.1057 & -0.2887 & 0.0000 & 0.1057 & 0.0000 \\
0.0774 & -0.2113 & 0.0000 & 0.0774 & 0.0000 \\
0.0000 & -0.0000 & 0.0000 & 0.0000 & 0.0000 \\
0.1057 & -0.2887 & 0.0000 & 0.1057 & 0.0000 \\
0.0000 & -0.0000 & 0.0000 & 0.0000 & 0.0000
\end{array}\right],
$$

and $\|\widehat{\Delta}\|_{2}=0.5176$. For this example, one can obtain the upper bound $\mu_{P D}^{\text {upper }}=1.9319$ while the same lower bound as $\mu_{P D}^{\text {lower }}=1.9319$. By making use of our algorithm [12], we have obtained the perturbation $\epsilon^{*} \Delta^{*}$ with

$$
\Delta^{*}=\left[\begin{array}{ccccc}
0.0000 & -0.0000 & -0.0000 & 0.0000 & 0.0000 \\
0.0000 & -0.0000 & -0.0000 & 0.0000 & 0.0000 \\
0.0000 & -0.0000 & -0.5000 & 0.0000 & 0.5000 \\
0.0000 & -0.0000 & -0.0000 & 0.0000 & 0.0000 \\
-0.0000 & 0.0000 & 0.5000 & -0.0000 & -0.5000
\end{array}\right],
$$

and $\epsilon^{*}=1.0000$ while $\left\|\Delta^{*}\right\|_{2}=1.0000$ The same lower bound can be obtained $\mu_{N e w}^{\text {lower }}=1.0000$ as the one obtained by mussv. 
Example 5. In the following table 7, we give comparison of numerical approximation to both lower and upper bounds of structured singular values approximated by the well-known MATLAB routine mussv and algorithm [12] for the matrix $A_{8}$. The matrix $A_{8}$ is gievn as below. In first column of table 7 , we present the size of the matrix $A_{8}$. While in the next column, we present the family of block diagonal matrices which is denoted by $\Theta_{\mathbb{B}}$. In the third, fourth and fifth columns we present both upper and lower bounds that is $\mu_{u}^{\text {mussv }}, \mu_{l}^{\text {mussv }}$ approximated by MATLAB routine mussv and the lower bound $\mu_{l}^{\text {New }}$ approximated by algorithm [12] respectively.

$$
A_{8}=\left[\begin{array}{ccccc}
0 & 0 & 1 & 1 & -1 \\
1 & -1 & 0 & 1 & 0 \\
0 & 1 & 1 & 0 & -1 \\
0 & 0 & 0 & 0 & 1 \\
0 & 0 & 1 & 0 & 0
\end{array}\right]
$$

In the following example, we consider a five dimensional complex matrix $B_{8}$ given as,

$$
B_{8}=\left[\begin{array}{ccccc}
-1 & 0 & 0 & 0 & 0 \\
0 & -1 & 0 & 0 & 0 \\
0 & 0 & -1 & 0 & 0 \\
1 & -1 & 0 & 1 & 0 \\
0 & -1 & -1 & 0 & 1
\end{array}\right]
$$

Consider the perturbation set as

$$
\Theta_{\mathbb{B}}=\left\{\operatorname{diag}\left(\Delta_{1}\right): \Delta_{1} \in \mathbb{C}^{5,5}\right\}
$$

By Applying the MATLAB function mussv, we have obtained the perturbation $\widehat{\Delta}$ with

$$
\widehat{\Delta}=\left[\begin{array}{ccccc}
-0.0288 & 0.0576 & 0.0288 & 0.1091 & 0.1091 \\
0.0576 & -0.1151 & -0.0576 & -0.2182 & -0.2182 \\
0.0288 & -0.0576 & -0.0288 & -0.1091 & -0.1091 \\
-0.0228 & 0.0455 & 0.0228 & 0.0863 & 0.0863 \\
-0.0228 & 0.0455 & 0.0228 & 0.0863 & 0.0863
\end{array}\right]
$$




\begin{tabular}{||l||c||c||c||c||}
\hline$n$ & $\Theta_{\mathbb{B}}$ & $\mu_{u}^{\text {mussv }}$ & $\mu_{l}^{\text {mussv }}$ & $\mu_{l}^{\text {New }}$ \\
\hline 05 & $\left\{\operatorname{diag}\left(\Delta_{1}\right): \Delta_{1} \in \mathbb{C}^{5,5}\right\}$ & 2.4142 & 2.4142 & 2.4142 \\
\hline 05 & $\left\{\operatorname{diag}\left(\delta_{i} I_{i}\right): \delta_{i} \in \mathbb{R}, \forall i=1: 5\right\}$ & 2.1616 & 2.1516 & 2.0609 \\
\hline 05 & $\left\{\operatorname{diag}\left(\delta_{i} I_{i}\right): \delta_{i} \in \mathbb{C}, \forall i=1: 5\right\}$ & 2.1628 & 2.1537 & 1.9511 \\
\hline 05 & $\left\{\operatorname{diag}\left(\delta_{1} I_{1}, \delta_{2} I_{1}, \Delta_{2}\right): \delta_{1}, \delta_{2} \in \mathbb{R}, \Delta_{1} \in \mathbb{C}^{3,3}\right\}$ & 2.2182 & 2.2174 & 2.2176 \\
\hline 05 & $\left\{\operatorname{diag}\left(\delta_{1} I_{1}, \delta_{2} I_{1}, \Delta_{2}\right): \delta_{1}, \delta_{2} \in \mathbb{C}, \Delta_{1} \in \mathbb{C}^{3,3}\right\}$ & 2.7178 & 2.7176 & 0.5000 \\
\hline 05 & $\left\{\operatorname{diag}\left(\Delta_{1}, \delta_{1} I_{1}, \Delta_{2}\right): \delta_{1} \in \mathbb{C}, \Delta_{1}, \Delta_{2} \in \mathbb{C}^{2,2}\right\}$ & 2.2592 & 2.2571 & 2.2589 \\
\hline 05 & $\left\{\operatorname{diag}\left(\Delta_{1}, \delta_{1} I_{1}, \Delta_{2}\right): \delta_{1} \in \mathbb{R}, \Delta_{1}, \Delta_{2} \in \mathbb{C}^{2,2}\right\}$ & 2.2592 & 2.2592 & 2.2592 \\
\hline
\end{tabular}

TABLE 7. Computation of bounds of $\mu$-values

and $\|\widehat{\Delta}\|_{2}=0.4569$. For this example, one can obtain the upper bound $\mu_{P D}^{u p p e r}=2.1889$ while the same lower bound as $\mu_{P D}^{\text {lower }}=2.1889$. By making use of our algorithm [12], one can obtain the perturbation $\epsilon^{*} \Delta^{*}$ with

$$
\Delta^{*}=\left[\begin{array}{ccccc}
-0.0630 & 0.1260 & 0.0630 & 0.2388 & 0.2388 \\
0.1260 & -0.2520 & -0.1260 & -0.4777 & -0.4777 \\
0.0630 & -0.1260 & -0.0630 & -0.2388 & -0.2388 \\
-0.0498 & 0.0997 & 0.0498 & 0.1890 & 0.1890 \\
-0.0498 & 0.0997 & 0.0498 & 0.1890 & 0.1890
\end{array}\right],
$$

and $\epsilon^{*}=0.4569$ while $\left\|\Delta^{*}\right\|_{2}=1.0000$. The same lower bound can be obtained $\mu_{\text {New }}^{\text {lower }}=2.1889$ as the one obtained by mussv.

\section{Conclusion}

In this article we have considered the numerical approximation of $\mu$-values for the matrix representations of finite symmetric groups $S_{n}$ over the filed of complex numbers by using well-known MATLAB function mussv and algorithm [12]. The experimental results indicates the different behaviors of lower bounds of $\mu$-values with once computed by mussv and our algorithm.

\section{REFERENCES}

[1] Bernhardsson, Bo and Rantzer, Anders and Qiu, Li. Real perturbation values and real quadratic forms in a complex vector space. Linear Algebra Appl., 1(1994): 131-154.

[2] Braatz, Richard P and Young, Peter M and Doyle, John C and Morari, Manfred. Computational complexity of $\mu$ calculation. IEEE Trans. Autom. Control, 39(1994): 1000-1002.

[3] Chen, Jie and Fan, Michael KH and Nett, Carl N. Structured singular values with nondiagonal structures. I. Characterizations. IEEE Trans. Autom. Control, 41(1996): 1507-1511.

[4] Danielson, Claus Robert. Symmetric constrained optimal control: Theory, algorithms, and applications. University of California, Berkeley, 2014. 
[5] Fan, Michael KH and Tits, André L and Doyle, John C. Robustness in the presence of mixed parametric uncertainty and unmodeled dynamics. IEEE Trans. Autom. Control, 36(1991): 25-38

[6] Hinrichsen, D and Pritchard, AJ. Mathematical systems theory I, vol. 48 of Texts in Applied Mathematics. SpringerVerlag, Berlin Volume 48(2005).

[7] Karow, Michael and Kokiopoulou, Effrosyni and Kressner, Daniel. On the computation of structured singular values and pseudospectra. Syst. Control Lett. 59(2010): 122-129.

[8] Karow, Michael and Kressner, Daniel and Tisseur, Françoise. Structured eigenvalue condition numbers. SIAM J. Matrix Anal. Appl. 28(2006): 1052-1068.

[9] Packard, Andrew and Doyle, John. The complex structured singular value. Automatica 29(1993): 71-109.

[10] Packard, Andy and Fan, Michael KH and Doyle, John. A power method for the structured singular value. Decision and Control, 1988., Proceedings of the 27th IEEE Conference on, 2132-2137, (1998).

[11] Qiu, Li and Bernhardsson, Bo and Rantzer, Anders and Davison, EJ and Young, PM and Doyle, JC. A formula for computation of the real stability radius. Automatica, 31(1995), 879-890.

[12] Rehman, Mutti-Ur and Tabassum, Shabana Numerical Computation of Structured Singular Values for Companion Matrices. J. Appl. Math. Phys., 5(2017), 1057-1072.

[13] Doyle, John Analysis of feedback systems with structured uncertainties. IEEE Proc. D-Control Theory Appl., 129(1982), $242-250$.

[14] Ferreres, Gilles A practical approach to robustness analysis with aeronautical applications. Springer Science $\mathcal{G}$ Business Media, (1999)

[15] Fu, Minyue The real structured singular value is hardly approximable. IEEE Trans. Autom. Control, 42(1997), 1286 1288.

[16] Newlin, Matthew P and Glavaski, Sonja T Advances in the computation of the/spl mu/lower bound. Proc. 1995 Amer. Control Conf., (1995). 